\title{
Factores relacionados con el inicio no programado de hemodiálisis en pacientes seguidos en consulta ERCA
}

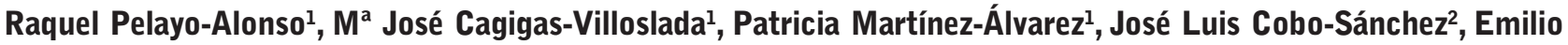 \\ Ibarguren-Rodríguez ${ }^{1}$, Rosa Ana Sáinz-Alonso ${ }^{1}$ \\ ${ }^{1}$ Servicio de Nefrología/Hemodiálisis. Hospital Universitario Marqués de Valdecilla. Santander. España \\ ${ }^{2}$ Área de Calidad, Formación, I+D+I de Enfermería. Hospital Universitario Marqués de Valdecilla. Santander. \\ España
}

Como citar este artículo: Pelayo-Alonso R, Cagigas-Villoslada MJ, Martínez-Álvarez P, Cobo-Sánchez JL, Ibarguren-Rodríguez E, Sáinz-Alonso RA. Factores relacionados con el inicio no programado de hemodiálisis en pacientes seguidos en consulta ERCA. Enferm Nefrol. 2020 Ene-Mar;23(1):68-74

\section{Resumen}

Introducción. El inicio programado del tratamiento renal sustitutivo es un objetivo prioritario en el manejo de los pacientes con enfermedad renal crónica, ya que supone un gran impacto para la supervivencia de estos pacientes.

Objetivo: Analizar características clínicas implicadas en el inicio no programado de la hemodiálisis en pacientes seguidos en consulta ERCA.

Material y Método: Estudio retrospectivo observacional en pacientes incidentes en el periodo 2014-2018. Se recogieron datos clínicos y sociodemográficos de la historia clínica del paciente, tiempo de seguimiento en consulta ERCA, filtrado glomerular al inicio de la consulta ERCA, causa y tipo de inicio (programado o no) de la hemodiálisis, así como el acceso vascular empleado.

Resultados: Se incluyeron 168 pacientes incidentes seguidos en consulta ERCA. EI 28,6\% inició hemodiálisis de forma no programada. Los inicios programados se debieron a causa urémica y los no programados, a insuficiencia cardíaca (92\% y 54\% respectivamente,

\section{Correspondencia:}

Raquel Pelayo Alonso raquel.pelayo@scsalud.es $p<0,001)$. Los pacientes con inicio no programado utilizaron un catéter en el $77 \%$ de las ocasiones $(p<0,001)$, tenían más edad $(69,27 \pm 9,4$ vs $65,18 \pm 12,75$ años) y un menor tiempo de seguimiento en la consulta ERCA $(15,60 \pm 12,37$ vs $23,64 \pm 20,25$ meses) que los pacientes con inicio programado.

Conclusiones: Pacientes de mayor edad, con menor tiempo de seguimiento en consulta ERCA tienen más riesgo de iniciar hemodiálisis de forma no programada a través de un catéter venoso central por falta de un acceso vascular definitivo.

PALABRAS CLAVE: enfermedad renal crónica avanzada; hemodiálisis; toma de decisiones compartidas; inicio no programado de diálisis.

\section{Factors related to unplanned dialysis start in patients followed in ESRD consultation}

\section{Abstract}

Introduction. The planned start of renal replacement therapy is a priority objective in the management of patients with chronic kidney disease, having a great impact on patient survival.

Objective: To analyse the clinical characteristics involved in the unplanned hemodialysis start in patients followed in ESRD consultation. 
Material and Method: Retrospective observational study in incident patients in the 2014-2018 period. Clinical and sociodemographic data were collected on the patient's medical record, follow-up time in the ESRD consultation, glomerular filtration at the beginning of the ESRD consultation, cause and type of hemodialysis start (planned or not), as well as the vascular access used.

Results: 168 incident patients followed in ESRD consultation were included. $28.6 \%$ started hemodialysis in an unplanned way. The planned dialysis starts were due to uremic cause and unplanned due to heart failure ( $92 \%$ and $54 \%$ respectively, $\mathrm{p}<0.001$ ). Patients with unplanned start used a catheter $77 \%$ of the time ( $p$ $<0.001)$, were older $(69.27 \pm 9.4$ vs. $65.18 \pm 12.75$ years) and shorter follow-up time in ESRD consultation ( $15.60 \pm 12.37$ vs. $23.64 \pm 20.25$ months) than patients with planned start.

Conclusions: Older patients, with shorter follow-up time in an ESRD consultation, have a higher risk of starting hemodialysis in an unplanned way through a central venous catheter due to lack of definitive vascular access.

KEYWORDS: advanced chronic kidney disease; hemodialysis; shared decision making; unplanned dialysis start.

\section{Introducción}

La Enfermedad Renal Crónica (ERC) es un importante problema de salud pública por su elevada incidencia, prevalencia y coste económico ${ }^{1,2}$. Según el Informe de Diálisis y Trasplante del Registro Español de Enfermos Renales de la Sociedad Española de Nefrología (SEN) ${ }^{3}$, la prevalencia del tratamiento renal sustitutivo (TRS) en el año 2017 se situaba en una tasa de 1284,2 ppm. De los pacientes incidentes en TRS, el 78\% inició el tratamiento mediante hemodiálisis y el $17,1 \%$ mediante diálisis peritoneal.

La SEN ${ }^{4}$ considera que el inicio programado del TRS debe ser un objetivo prioritario en los pacientes con ERC ya que supone un gran impacto sobre la supervivencia de estos pacientes ${ }^{4-6}$. Se entiende por inicio programado (IP) o inicio óptimo cuando el paciente inicia tratamiento dialítico de manera electiva en un entorno ambulatorio, con un catéter peritoneal o fístula arteriovenosa madura y en la modalidad de diálisis crónica elegida por el paciente ${ }^{7}$. Pese a las recomendaciones, los inicios no programados (INP) son frecuentes en las unidades de hemodiálisis, con tasas cercanas al 50\% según la bibliografía ${ }^{4,8}$. Estos INP se traducen en mayor morbilidad y mortalidad y peor calidad de vida para los pacientes ${ }^{9}$.

Esta situación ha generado, que las directrices actuales recomienden la remisión temprana del paciente con ERC al nefrólogo ${ }^{1}$ y la puesta en marcha de consultas de enfermedad renal crónica avanzada (ERCA) multidisciplinares que incluyan nefrólogos, enfermeras nefrológicas o dietistas, entre otros, para abordar de forma integral aspectos relativos al tratamiento de estos pacientes, cuyos objetivos deben ser disminuir y tratar complicaciones y preparar de forma adecuada y con suficiente antelación el inicio del TRS evitando así inicios no programados $2,10,11$.

\section{Objetivo}

Analizar posibles características clínicas implicadas en el inicio no programado de hemodiálisis crónica de los pacientes seguidos en consulta ERCA.

\section{Material y Método}

Se realizó un estudio retrospectivo observacional sobre pacientes que iniciaron TRS con hemodiálisis crónica entre el 1 de enero de 2014 y el 31 de diciembre de 2018 y que habían sido seguidos en la consulta ERCA del Servicio de Nefrología del Hospital Universitario Marqués de Valdecilla (de referencia para la Comunidad Autónoma de Cantabria), al menos 1 mes antes de la entrada a diálisis. Se excluyeron a los pacientes que iniciaron HD crónica en los que no se realizó seguimiento nefrológico previo al inicio del tratamiento. Se recogieron datos clínicos y sociodemográficos de la histórica clínica electrónica del paciente, incluyendo la edad, sexo, nefropatía de base, comorbilidad según el índice de Charlson. Además, se recogieron antecedentes personales de diabetes, hipertensión arterial, patología cardiovascular, grado de obesidad según el Índice de Masa Corporal (IMC) y el tratamiento domiciliario anterior al inicio de la hemodiálisis. Otras variables a estudio fueron el tiempo de seguimiento en la consulta ERCA; el filtrado glomerular al inicio del seguimiento en la consulta ERCA (según la fórmula CKD-EPI); tipo de inicio de hemodiálisis: programado (IP) o no programado (INP). 
Entre los INP se evaluó la necesidad de ingreso hospitalario y hemodiálisis urgente o con una demora máxima de 24 horas; la causa de inicio de la diálisis (uremia; insuficiencia cardíaca; uremia e insuficiencia cardiaca; secundaria a otro problema de salud); y el acceso vascular (AV) de inicio empleado.

El estudio se llevó a cabo cumpliendo la Ley Orgánica $3 / 2018$, de 5 de diciembre de protección de datos personales y garantía de los derechos digitales.

A partir de las variables estudiadas, se elaboró una base de datos tipo Excel. Para el análisis de los datos se utilizó el paquete estadístico PSPP v1.2. Se realizó análisis descriptivo utilizando medidas de tendencia central para las variables cuantitativas y frecuencias y porcentajes para las variables cualitativas. Para la inferencia estadística se utilizaron los test $\mathrm{Chi}^{2}, \mathrm{~T}$ de Student y contraste bilateral, considerándose un valor $\mathrm{p}<0,05$ como estadísticamente significativo.

\section{Resultados}

Durante el periodo de estudio, comenzaron tratamiento con hemodiálisis crónica 241 pacientes, de los cuales se incluyeron en el presente estudio los 168 pacientes que habían tenido seguimiento nefrológico en la consulta ERCA.

La edad media de la muestra fue de $66,35 \pm 12$ años (rango 27-86). El 73,2\% fueron hombres y según el Índice de Charlson, la comorbilidad media fue de $4,52 \pm 1,87$ puntos (rango 2-10). El filtrado glomerular medio al inicio de la consulta ERCA fue de $17,23 \pm 4,79$ $\mathrm{ml} / \mathrm{ml} / 1,73 \mathrm{~m}^{2}$ (rango 6-26), con un tiempo medio de seguimiento en consulta ERCA de $21,35 \pm 18,76$ meses (rango 1-120). En la Tabla 1 se detallan las principales características clínicas de los pacientes.

El 28,6\% de los pacientes inició hemodiálisis de forma no programada. En la Tabla 2 se muestran diferentes variables clínicas relacionadas según el tipo de inicio de hemodiálisis. El motivo más frecuente de INP fue la insuficiencia cardiaca, en el $54 \%$ de los casos, mientras que la uremia fue responsable del $92 \%$ de los IP $(p<0,01)$.

El acceso vascular de inicio utilizado por los pacientes con IP fue la fístula arteriovenosa nativa (FAVn), usado en el $71 \%$ de los casos, mientras que, en el INP, el catéter venoso central (CVC), tanto tunelizado como no tunelizado, fue el AV de elección en el $77 \%$ de las ocasiones $(p<0,01)$.
Tabla 1. Características clínicas de los pacientes incidentes en hemodiálisis crónica en el periodo de estudio.

\begin{tabular}{|c|c|c|}
\hline & $\mathbf{n}$ & $\%$ \\
\hline \multicolumn{3}{|l|}{ Sexo } \\
\hline - Hombre & 123 & $73,2 \%$ \\
\hline - Mujer & 45 & $26,8 \%$ \\
\hline \multicolumn{3}{|l|}{ Nefropatía de base } \\
\hline - Diabética & 49 & $29,2 \%$ \\
\hline - Vascular & 40 & $23,8 \%$ \\
\hline - Glomerular & 29 & $17,3 \%$ \\
\hline - Intersticial & 12 & $7,1 \%$ \\
\hline - Sistémica & 4 & $2,4 \%$ \\
\hline - Congénita & 14 & $8,3 \%$ \\
\hline - Otras & 20 & $11,9 \%$ \\
\hline \multicolumn{3}{|c|}{ Antecedentes personales } \\
\hline - Diabetes Mellitus & 80 & $47,6 \%$ \\
\hline - Hipertensión Arterial & 151 & $89,9 \%$ \\
\hline - Cardiopatía & 47 & $28 \%$ \\
\hline \multicolumn{3}{|c|}{ Grado de obesidad (según IMC) } \\
\hline - Bajo peso & 2 & $1,2 \%$ \\
\hline - Normopeso & 51 & $30,4 \%$ \\
\hline - Sobrepeso & 57 & $33,9 \%$ \\
\hline - Obesidad & 58 & $34,6 \%$ \\
\hline \multicolumn{3}{|l|}{ Tratamiento domiciliario } \\
\hline - Anti-HTA & 135 & $80,4 \%$ \\
\hline - Diuréticos & 125 & $74,4 \%$ \\
\hline - Bicarbonato & 72 & $42,9 \%$ \\
\hline \multicolumn{3}{|c|}{ Motivo de inicio de Hemodiálisis } \\
\hline - Uremia & 123 & $73,2 \%$ \\
\hline - IC & 27 & $16,1 \%$ \\
\hline - Uremia+IC & 13 & $7,7 \%$ \\
\hline $\begin{array}{l}-2^{\mathrm{a}} \text { a otro problema } \\
\text { de salud }\end{array}$ & 5 & $3 \%$ \\
\hline
\end{tabular}

IMC: Índice de Masa Corporal; IC: Insuficiencia Cardiaca.

En el grupo de pacientes con INP se observó un mayor porcentaje de diabéticos que en el grupo de pacientes con IP (diabetes $62,5 \%$ vs no diabetes $42 \% ; p=0,015$ ). En cuanto al tratamiento domiciliario, el $89,5 \%$ de los pacientes con INP seguían tratamiento con diurético frente el $68 \%$ del grupo de IP $(p=0,04)$.

Otros factores implicados se especifican en la Tabla 3. Los pacientes con INP presentaron mayor edad (INP $69,27 \pm 9,40$ años vs IP $65,18 \pm 12,75$ años; $p=0,046$ ); mayor tasa de filtrado glomerular en el momen- 
Tabla 2. Variables clínicas relacionadas según el tipo de inicio de hemodiálisis.

\begin{tabular}{|c|c|c|c|c|c|c|}
\hline \multicolumn{2}{|l|}{ PARÁMETRO } & \multicolumn{2}{|c|}{$\operatorname{IP}(n$ 120) } & \multicolumn{2}{|c|}{ INP (n 48) } & \multirow[t]{2}{*}{$\mathbf{p}$} \\
\hline & & $\%$ & $\mathbf{n}$ & $\%$ & $\mathbf{n}$ & \\
\hline \multirow[t]{2}{*}{ Sexo } & Hombre & $74 \%$ & 89 & $70 \%$ & 34 & \multirow[t]{2}{*}{ NS } \\
\hline & Mujer & $26 \%$ & 31 & $30 \%$ & 14 & \\
\hline \multirow[t]{2}{*}{ Acceso Vascular } & FAVn & $71 \%$ & 85 & $23 \%$ & 11 & \multirow[t]{2}{*}{$p<0,01$} \\
\hline & CVC & $29 \%$ & 35 & $77 \%$ & 37 & \\
\hline \multirow[t]{4}{*}{ Motivo de inicio en Hemodiálisis crónica } & Uremia & $92 \%$ & 110 & $27 \%$ & 13 & \multirow[t]{4}{*}{$p<0,01$} \\
\hline & IC & - & - & $54 \%$ & 26 & \\
\hline & Uremia+IC & $8 \%$ & 10 & $8 \%$ & 4 & \\
\hline & $2^{\mathrm{a}} \mathrm{a}$ otras causas & - & - & $11 \%$ & 5 & \\
\hline \multicolumn{2}{|l|}{ AP previo de Diabetes Mellitus } & $42 \%$ & 50 & $62,5 \%$ & 30 & 0,015 \\
\hline \multicolumn{2}{|l|}{ AP previo de HTA } & $89 \%$ & 107 & $92 \%$ & 44 & NS \\
\hline \multicolumn{2}{|l|}{ AP previo de cardiopatía } & $29 \%$ & 35 & $25 \%$ & 12 & NS \\
\hline \multicolumn{2}{|l|}{ Obesidad (IMC>30) } & $35 \%$ & 42 & $33 \%$ & 16 & NS \\
\hline \multicolumn{2}{|l|}{ Tratamiento con anti-HTA } & $80 \%$ & 37 & $77 \%$ & 96 & NS \\
\hline \multicolumn{2}{|l|}{ Tratamiento con diurético } & $68 \%$ & 82 & $89,5 \%$ & 43 & 0,04 \\
\hline \multicolumn{2}{|l|}{ Tratamiento con Bicarbonato } & $45 \%$ & 54 & $37,5 \%$ & 18 & NS \\
\hline
\end{tabular}

IP: Inicio Programado; INP: Inicio No Programado. NS: no significativo ( $p>0.05$ ); FAVn: Fístula Arteriovenosa Interna nativa; CVC: Catéter Venoso Central; IC: Insuficiencia Cardiaca; IMC: Índice de Masa Corporal.

Tabla 3. Valores medios y desviación típica (DE) de variables clínicas según la forma de inicio de la hemodiálisis.

\begin{tabular}{|c|c|c|c|c|c|}
\hline \multirow[t]{2}{*}{ PARÁMETRO } & \multicolumn{2}{|c|}{ IP } & \multicolumn{2}{|c|}{ INP } & \multirow[t]{2}{*}{$\mathbf{p}$} \\
\hline & Media & DE & Media & DE & \\
\hline Edad (años) & 65,18 & 12,75 & 69,27 & 9,40 & 0,046 \\
\hline Filtrado Glomerular al inicio ERCA $\left(\mathrm{ml} / \mathrm{min} / 1,73 \mathrm{~m}^{2}\right)$ & 16,62 & 4,713 & 18,76 & 4,71 & 0,01 \\
\hline Seguimiento consulta ERCA (meses) & 23,64 & 20,55 & 15,60 & 12,37 & 0,011 \\
\hline IC Charlson (puntos) & 4,10 & 1,62 & 5,56 & 1,98 & $<0,01$ \\
\hline IMC $\left(\mathrm{kg} / \mathrm{m}^{2}\right)$ & 28,40 & 5,49 & 28,15 & 5,48 & NS \\
\hline
\end{tabular}

IP: Inicio Programado; INP: Inicio No Programado. NS: no significativo ( $p>0.05$ ); FAVn: Fístula Arteriovenosa Interna nativa; CVC: Catéter Venoso Central; IC: Insuficiencia Cardiaca; IMC: Índice de Masa Corporal.

to de inicio de la consulta ERCA (INP 18,76 $\pm 4,71$ $\mathrm{ml} / \mathrm{min} / 1,73 \mathrm{~m}^{2}$ vs IP $16,62 \pm 4,73 \mathrm{ml} / \mathrm{min} / 1,73 \mathrm{~m}^{2}$; $\mathrm{p}=0,01$ ); un menor tiempo de seguimiento nefrológico en la consulta ERCA (INP 15,60 $\pm 12,37$ meses vs IP $23,64 \pm 20,55$ meses; $p=0,011$ ) y una mayor comorbilidad que los pacientes con un IP (INP $5,56 \pm 1,98$ vs IP $4,10 \pm 1,62 ; p<0,001)$.

\section{Discusión}

EI IP del TRS tiene un impacto positivo en la supervivencia del paciente ${ }^{7,8}$. Diversos estudios $1,5,7,12$ refieren que la principal causa de los INP de la diálisis se relaciona con la remisión tardía al nefrólogo. Las guías clínicas ${ }^{4}$ indican que todos los pacientes con ERC en estadio IV deben ser remitidos al nefrólogo para su valoración. Sin embargo, según nuestros datos, los pacientes tuvieron una remisión tardía, con tasas de filtrado glomerular medio de $17,23 \pm 4,79$ $\mathrm{ml} / \mathrm{min} / 1,73 \mathrm{~m}^{2}$. No obstante, los pacientes con INP presentaron una mayor tasa de filtrado glomerular al inicio de la consulta ERCA que los pacientes con IP. Caravaca y cols. ${ }^{6}$ determinan que una correcta atención durante la fase de prediálisis mediante IECAs y ARAII para el control de la HTA y el uso de bicarbonato sódico para corregir la acidosis metabólica, tiene efectos favorables sobre el mantenimiento de 
la función renal permitiendo retrasar la necesidad de diálisis. En nuestra muestra, los pacientes en tratamiento con estos anti-hipertensivos y con bicarbonato sódico son más frecuentes en el grupo de IP que en los de INP, lo que puede explicar, que pese a una menor función renal a su llegada a ERCA, ésta se mantiene durante más tiempo, favoreciendo los IP. El retraso en la remisión al nefrólogo se relaciona además con tasas de inicios subóptimos entorno al $50 \%$ según las series $5,12,13$. En nuestra muestra, la tasa de INP fue del $28,6 \%$, aunque todos nuestros pacientes procedían de la consulta ERCA, al igual que en la muestra de Lorenzo y cols. ${ }^{8}$ en cuyo caso, la tasa de INP alcanza el $55 \%$ de los pacientes incidentes. Lo que sí tienen en común ambas series es que los pacientes con INP presentan una mayor edad y utilizan con mayor frecuencia, un CVC como acceso vascular de inicio. En relación con este aspecto, mencionar que estudios previos indican que el INP de hemodiálisis supone utilizar un CVC como acceso vascular ${ }^{5-7,14}$ lo que aumenta el riesgo de mortalidad, hospitalizaciones y costes económicos, más aún en pacientes más ancianos ${ }^{15}$, grupo de edad que mayor tasa de INP presenta. Guías de práctica clínica reconocidas ${ }^{16,17}$ recomiendan la preparación previa del AV con tiempo suficiente para iniciar la hemodiálisis con una FAVn madura. Sin embargo, pese a que los pacientes de nuestra muestra proceden de la consulta ERCA, el $77 \%$ de los pacientes con INP y el $29 \%$ de los pacientes con IP, iniciaron HD a través de un CVC. Este mismo comportamiento se produce en otras series consultadas ${ }^{5,7,12}$, en las que un alto porcentaje de pacientes inician HD sin un AV definitivo. Este retraso en la disponibilidad de un $\mathrm{AV}$ definitivo pese a la remisión al nefrólogo puede estar relacionado con un retraso en la toma de decisiones por parte de los pacientes ${ }^{5}$. Es necesario fomentar la creación y el impulso de la Unidades de ERCA con presencia no sólo de nefrólogos, sino también de enfermeras nefrológicas. Forero y cols. ${ }^{18}$ en su revisión sistemática ponen de manifiesto que una mayor atención del paciente antes de su entrada en diálisis en las consultas de Enfermería de ERCA permite individualizar cuidados, proporcionar información completa encaminada a favorecer la toma de decisiones sobre las distintas modalidades de TRS y modificar hábitos de vida que permiten fomentar autocuidados disminuyendo complicaciones. Esta actuación facilita la toma de decisiones del paciente permitiendo dar prioridad a la preparación requerida en función de la opción de TRS escogida por el paciente ${ }^{8}$, es decir, realizar FAVn si el paciente escoge hemodiálisis o colocar un catéter pe- ritoneal si la opción escogida es la diálisis peritoneal, con el objetivo final de disminuir los inicios no programados por falta de acceso definitivo ${ }^{10}$. Para ello es importante adecuar el tiempo de preparación para iniciar TRS a cada institución sanitaria en función de las listas de espera, de tal forma que el paciente pueda disponer de una FAVn o un catéter peritoneal funcionante en el momento que sea necesario iniciar el tratamiento dialítico.

La principal causa de INP de hemodiálisis en nuestro estudio es la insuficiencia cardíaca, presente en el 54\% de los casos. La insuficiencia cardiaca es una complicación frecuente en al ERC, más aún en pacientes ERCA que aún no han iniciado diálisis, lo que puede explicar también por qué es más frecuente el tratamiento con diuréticos en pacientes con INP que en aquellos que presentaron un IP. Martínez y cols. ${ }^{19}$ demuestran esta misma tendencia, siendo la insuficiencia cardiaca responsable del $63 \%$ de los INP frente al $3 \%$ de los IP de su serie. Desde las consultas ERCA puede ser interesante implantar intervenciones como el correcto manejo nutricional, restricción hídrica, control de composición corporal mediante bioimpedancia o la correcta adherencia al tratamiento con el objetivo final de prevenir esta complicación.

Otra característica clínica que nos encontramos en los pacientes con INP de nuestro estudio es la presencia de diabetes, más frecuente que en el grupo de pacientes con IP. Un reciente estudio ${ }^{20}$ de ámbito nacional sobre pacientes diabéticos mayores de 65 años establece que la prevalencia de la ERC fue del $37,2 \%$ y que esta tasa aumentaba con la edad. Según el Informe de Diálisis y Trasplante del Registro Español de Enfermos Renales de la Sociedad Española de Nefrología3 ${ }^{3}$, en el año 2017 la principal causa de ERC fue la diabetes, responsable del $23,9 \%$ del total de las nefropatías.

Además de la diabetes, otras características que definen al paciente con INP son una mayor edad, un menor tiempo de seguimiento en la consulta ERCA y una mayor comorbilidad asociada, misma situación que objetivan Gorritz y cols. ${ }^{14}$ cuando comparan los pacientes con IP y pacientes con INP de su estudio.

Los datos de este estudio refrendan que la valoración de la comorbilidad se presenta como necesaria en las consultas ERCA. El índice de Charlson es un instrumento útil y de práctica sencilla para enfermería y debería implementarse como instrumento necesario en las consultas de enfermería de ERCA ${ }^{21}$. 
No era objetivo del estudio analizar las tasas de supervivencia y/o mortalidad relacionadas con el tipo de inicio de hemodiálisis, aunque sin duda alguna, sería una variable a considerar, como ya lo han demostrado estudios previos. Se debe considerar el analizar de forma periódica las tasas de INP en las Unidades de Hemodiálisis con el objetivo de establecer áreas de mejora.

Como conclusión, en función de nuestros datos, los pacientes de mayor edad, con un mayor grado de comorbilidad, diabéticos y un menor tiempo de seguimiento en la consulta ERCA tienen mayor riesgo de comenzar hemodiálisis de forma no programada. El acceso vascular más frecuente en el inicio no programado es el catéter venoso central.

Se deben tener en cuenta estos factores para diseñar las intervenciones más adecuadas a realizar en las consultas ERCA con el objetivo de disminuir los inicios de hemodiálisis no programados.

Recibido: 15-11-19

Revisado: 05-12-19

Modificado: 28-01-20

Aceptado: 10-02-20

\section{Bibliografía}

1. Magalhaes LP, Dos Reis LM, Graciolli FG, Pereira BJ, de Oliveira RB, de Souza AA et al. Predictive factors of one-year mortality in a cohort of patients urdergoing urgent-start hemodialysis. PLoS One 2017;12(1):e0167895.

2. Alcázar R, Orte L, Otero A. Enfermedad renal crónica avanzada. Nefrologia. 2008; (Supl 3):S3-6.

3. Informe de Diálisis y Trasplante. Registros autonómicos de enfermos renales. Sociedad Española de Nefrología. Disponible en: https://www.senefro.org/ contents/webstructure/Informe_REER_2017.pdf.

4. Gomis $A$, Teruel JL, Fernández $M$, Rivera $M$, Rodríguez $N$, Jiménez $S$, et al. Causas de inicio no programado del tratamiento renal sustitutivo con hemodiálisis. Nefrología 2011;31(6):733-7.

5. Hughes SA, Mendelssohn JG, Tobe SW, McFarlane PA, Mendelssohn DC. Factors associated with suboptimal initiation of dialysis despite early nephrologist referral. Nephrol Dial Transplant 2013;28(2):392-7.

6. Caravaca F, Alvarado R, García-Pino G, Martínez-Gallardo $R$, Luna $E$. ¿Qué intervenciones terapéuticas durante el estadio prediálisis de la enfermedad renal crónica se asocian a una mejor supervivencia? Nefrología 2014;34(4):469-76.

7. Mendelssohn DC, Malmberg C, Hamandi B. An integrated review of "unplanned" dialysis initiation: reframing the terminology to "suboptimal" initiation. BMC Nephrol 2009;10:22.

8. Lorenzo V. Análisis de la frecuentación de Urgencias en consulta ERCA (enfermedad renal crónica avanzada): enseñanzas para optimizar el inicio programado en tratamiento renal sustitutivo. Nefrología 2018;38(6):622-9.

9. Selgas R, Aguilar J, Julián JC, Toledo A. Realidad y futuro en el tratamiento de la enfermedad renal crónica avanzada (ERCA). Nefrología 2007; 27(6):689-93.

10. Sánchez JA. Planificación anticipada e inicio de diálisis. Nefrología 2009;29(4):285-7.

11. Cirera F. Influencia de la consulta de acogida en diálisis sobre la elección del tratamiento renal sustitutivo. Enferm Nefrol 2016;19(3):242-7.

12. Mendelssohn DC, Curtis B, Yeates $K$, Langlois $S$, MacRae JM, Semeniuk LM, et al. Suboptimal initiation of dialysis with and without early referreal to a nephologist. Nephrol Dial Transplant 2011; 26:2959-65.

13. Azevedo LM, Ruiz-Calero RM, Chávez E, González B, Bayo MA, Barroso S, et al. ¿Cómo inician hemodiálisis los pacientes en nuestro hospital? Diálisis Traspl. 2015;36(2):60.

14. Górriz JL, Sancho A, Pallardó $L M$, Amoedo $M L$, Martín M, Sanz P, et al. Significado pronóstico de la diálisis programada en pacientes que inician tratamiento sustitutivo renal. Un estudio multicéntrico español. Nefrología 2002;22(1):49-59. 
15. Ocak G, Halbesma N, le Cessie S, Hoogeveen EK, van Dijk S, Hooman J, et al. Haemodialysis catheters increase mortality as compared to arteriovenous accesses especially in elderly patients. Nephrol Dial Transplant 2011;26(8):2611-7.

16. National Kidney Foundation KDOQUI Clinical Practice Guidelines for Hemodialysis Adequacy 200. Am J Kidney Dis 2001;37:557-64.

17. Guía Clínica Española del Acceso Vascular para Hemodiálisis. Enferm Nefrol 2018;21(Supl1).

18. Forero J, Barrios S. Rol de enfermería en la consulta de prediálisis en el paciente con enfermedad renal crónica avanzada. Enferm Nefrol 2016; 19(1):77-86.

19. Martínez R, Ferreira F, García G, Cerezo I, Hernández $R$, Caravaca $F$. Insuficiencia cardiaca en la enfermedad renal crónica avanzada: relación con el acceso vascular. Nefrología 2012;32(2):206-12.
20. Martínez J, Sangrós J, García FJ, Millaruelo JM, Díez J, Bordonaba D, et al. Enfermedad renal crónica en España: prevalencia y factores relacionados en personas con diabetes mellitus mayores de 64 años. Nefrología 2018;38(4):401-13.

21. Álvarez G, Nogueira A, Moral MA, Barril G. Utilidad del índice de comorbilidad de Charlson y del índice de Barthel en la valoración a pacientes en las unidades de enfermedad renal crónica avanzada (ERCA). Enferm Nefrol 2018;21(Supl 2):S335.

Este artículo se distribuye bajo una Licencia Creative Commons Atribución-NoComercial 4.0 Internacional. https://creativecommons.org/licenses/by-nc/4.0/

Open Access (c) (1) (8) 